\title{
AFRONTAMIENTO RESILIENTE Y MIEDO A PERDERSE ALGO EN TIEMPOS DE PANDEMIA POR COVID-19: UN ESTUDIO PILOTO SOBRE EL PAPEL MODERADOR DE LA REGULACIÓN EMOCIONAL
} RESILIENT COPING AND FEAR OF MISSING OUT IN TIMES OF COVID-19 PANDEMIC: A PILOT STUDY ABOUT THE MODERATING ROLE OF EMOTIONAL REGULATION

Laura Bandera-Pastor Facultad de Psicología (Universidad de Málaga)

Cirenia Quintana-Orts Facultad de Psicología (Universidad de Sevilla)

Lourdes Rey Facultad de Psicología (Universidad de Málaga)

Cómo citar este texto:

Bandera-Pastor, L., Quintana-Orts, C. y Rey, L. (2022). Afrontamiento resiliente y miedo a perderse algo en tiempos de pandemia por Covid-19: un estudio piloto sobre el papel moderador de la regulación emocional. Health and Addictions / Salud y Drogas, 22(1), 253-267. doi: 10.21134/haaj.v22i1.679 


\title{
Resumen
}

El periodo de pandemia por Covid-19 ha supuesto un fuerte impacto en la vida de las personas, asociándose a fenómenos problemáticos, entre ellos, el FoMO (Fear of Missing Out o miedo a perderse algo). Este miedo ha sido relacionado con numerosos problemas psicológicos, tanto internalizantes como externalizantes. Sin embargo, hasta la fecha, pocos estudios se han centrado en conocer aquellas variables que pueden ejercer un papel protector ante este miedo. El objetivo del presente estudio fue explorar el potenciar papel protector que podrían ejercer el afrontamiento resiliente y la regulación emocional conjuntamente, como recursos personales frente al FoMO durante la situación de pandemia. Una muestra de 146 jóvenes adultos de entre 18 y 25 años (74\% mujeres) cumplimentaron distintos cuestionarios que evaluaban: afrontamiento resiliente (BRS), regulación emocional (WLEIS-S) y miedo a perderse algo (FoMO). Se realizaron análisis de correlación, así como de moderación mediante la macro de PROCESS (Hayes, 2018). Los resultados señalaron asociaciones significativas entre las variables en la dirección esperada. Además, la regulación emocional moderó el efecto principal del afrontamiento resiliente sobre el FoMO. Estos hallazgos sugieren los potenciales beneficios de entrenar y desarrollar ambos recursos personales con el fin de protegerse de emociones negativas como el FoMO.

\begin{abstract}
The Covid-19 pandemic period has had a strong impact on people's lives, it has been associated with problematic phenomena, including FoMO (Fear of Missing Out). This fear has been related to numerous psychological problems, both internalizing and externalizing. However, to date, few studies have focused on variables that can play a protective role to deal with this fear. The objective of the present study was to explore the protective role that Resilient Coping and Emotional Regulation could play as personal resources against FoMO. A sample of 146 young adults, aged between 18 and 25 years (74\% women), completed different questionnaires regarding resilient coping (BRS), emotional regulation (WLEIS-S) and fear of missing out (FOMO). Correlation analysis was carried out among the variables, as well as a moderation analysis using the PROCESS macro (Hayes, 2018). The results indicated significant associations between the variables in the expected direction. In addition, Emotional Regulation moderated the main effect of Resilient Coping on FoMO. These findings suggest the potential benefits of training and developing both personal resources to protect against negative emotions such as FoMO.
\end{abstract}

\section{Palabras clave}

Miedo a Perderse Algo, FoMO, Regulación Emocional, Afrontamiento Resiliente, Pandemia.

\section{Keywords}

Fear of Missing Out, FoMO, Emotional Regulation, Resilient Coping, Pandemic. 


\section{INTRODUCCIÓN}

La Organización Mundial de la Salud (OMS) declaró, el 11 de marzo de 2020, el estado de pandemia por la enfermedad causada por el Covid-19. Esta enfermedad ha afectado a más de 185 países alrededor del mundo (Dong et al., 2020). Con el fin de proteger la salud pública, reforzar el sistema sanitario y contener la progresión exponencial del virus, el 14 de marzo de 2020, el Gobierno de España aprobó un Real Decreto (RD 463/2020) por el que se establecía un estado de alarma (Lasa et al., 2020), que se ha ido extendiendo hasta la actualidad. A lo largo de todo este periodo, la población española ha atravesado diferentes escenarios que van desde un estricto confinamiento domiciliario, pasando por una desescalada, hasta alcanzar una "nueva normalidad" que incluye una serie de restricciones a la movilidad y a la vida social que varían continuamente en función de la evolución del virus en cada región. Esta serie de circunstancias han provocado un gran cambio en la vida diaria de las personas, tanto por las medidas que se adoptan para evitar el contagio, como por sus efectos asociados en la salud física, psicológica, comportamientos y formas de interaccionar (Lima et al., 2020).

Si nos centramos en el ámbito psicológico, diferentes estudios alertan del impacto de la pandemia en esta esfera. Un estudio realizado por Parrado-González y León-Jariego (2020), en el que participaron 1596 sujetos españoles, catalogó el impacto psicológico del periodo de pandemia de moderado a severo en el $24.7 \%$ de los participantes, y apuntó a un deterioro en la salud mental en el $48.8 \%$ de los casos. Por su parte, Sandín et al. (2020) en su estudio con 1161 participantes españoles, evidenciaron el fuerte impacto emocional de este periodo, que se reflejó en problemas de sueño, miedos (a la enfermedad/contagio/muerte, a los problemas laborales o de ingresos, y al aislamiento social), y sintomatología emocional (estrés, preocupación, depresión, desesperanza, ansiedad, inquietud y nerviosismo). A su vez, a nivel comportamental, el periodo de pandemia ha sido fuertemente asociado a un mayor uso de las nuevas tecnologías, tanto con fines sociales como de ocio (Ariza et al., 2021; Dong et al., 2020).

Si bien es cierto que el uso de este tipo de servicios durante esta etapa ha podido facilitar experiencias positivas como son el entretenimiento, la necesidad de información o el sobrellevar el estrés (Polizzi et al., 2020), la literatura también ha evidenciado que este tipo de actividades online pueden suponer una amenaza para el desarrollo de otro tipo de consecuencias nocivas, como puede ser su uso problemático o abusivo, problemas de depresión o de ansiedad (Gao et al., 2020). En este sentido, diversos estudios se han centrado en conocer qué variables, más allá del aislamiento social percibido en este periodo de pandemia, podría haber tras el incremento en el uso de internet y redes sociales. En esta línea, el miedo a perderse algo (en inglés, Fear of Missing Out, FoMO) se ha postulado como una de las variables que podrían explicar este aumento (Fumagalli et al., 2021; Gioia et al., 2021; Hayran et al., 2021).

El FoMO es definido como una aprensión a que otras personas del entorno social extenso puedan estar teniendo experiencias gratificantes en las que uno está ausente (Przybylski et al., 2013). En términos más generales, se consideraría como un sentimiento negativo que emerge como resultado de ser conscientes de las propias experiencias desatendidas (Przybylski et al., 2013). Una posible explicación al incremento de este fenómeno en los últimos años ha sido relacionada con el auge que ha experimentado el uso de redes sociales y herramientas digitales que hace que, a menudo, las personas se vean atrapadas en un sentimiento de que otras personas están experimentando momentos más agradables y tienen una vida mejor (Hayran et al., 2021).

Con las medidas de cuarentena implementadas desde el inicio de la pandemia, que han conllevado una ausencia de fotos de viajes, fiestas o actividades placenteras en redes sociales, podría parecer que se produciría una reducción de los niveles de FoMO en la población. Sin embargo, tal y como se ha apuntado anteriormente, el FoMO se ha seguido produciendo a lo largo de este periodo, postulándose como una de las causas de un mayor uso de nuevas tecnologías. Así, Fumagalli et al. (2021), en su estudio analizaron diferentes variables que podrían relacionarse con el incremento del uso de redes sociales en un grupo de 334 jóvenes adultos. Entre sus resultados hallaron que este mayor uso desde el inicio de la pandemia podría estar relacionado con sentimientos de aislamiento y soledad, relación que podría estar fuertemente mediada por el miedo a perderse algo. Por su parte, en la línea del estudio anterior, Gioia et al. (2021), analizando los resultados de 487 adultos, encontraron que los niveles de FoMO podrían haber fortalecido conductas dirigidas a la comunicación en línea, lo que, a su vez, podría haber puesto a algunos participantes en riesgo de un uso problemático de redes sociales. En línea con los anteriores, otro estudio aportó resultados similares al señalar que el FoMO durante el periodo de pandemia no ha desaparecido, sino que ha sido 
transformado hacia un tipo de miedo a perderse algo relacionado con actividades online (Hayran et al., 2021). Así, es tentativo pensar que el FoMO puede haber sido una consecuencia no deseable en estos tiempos de estrés como el vivido a lo largo de este periodo de pandemia por Covid-19.

Atendiendo a los datos evidenciados sobre la presencia de este fenómeno a lo largo de este periodo, parecería interesante conocer las consecuencias para el bienestar psicológico que este miedo a perderse algo puede suponer en población joven. En este sentido, el FoMO ha sido asociado a diferentes problemas psicológicos y de salud. Por ejemplo, se ha considerado que puede conducir a problemas de ansiedad y estrés (Beyens et al., 2016), problemas de sueño (Adams et al., 2017), depresión (Baker et al., 2016), uso problemático de smartphones y redes sociales (Elhai et al., 2015; Wegmann et al., 2017) y a desmotivación académica (Alt, 2015). A su vez, el FoMO ha sido asociado a relaciones sociales pobres (Przyblski et al., 2013), a menores niveles de autoestima (Buglass et al., 2017) y a una menor satisfacción con la vida (Błachnio \& Przepiórka, 2018).

En contraposición al FoMO como consecuencia indeseable derivada de este periodo, se han encontrado otros factores, como es el afrontamiento resiliente, con potencial protector ante eventos vitales estresantes y sus consecuencias negativas asociadas. La resiliencia ha sido definida como un proceso dinámico a través del cual los individuos experimentan una adaptación positiva ante situaciones difíciles, de adversidad, o ante traumas (Luthar \& Cicchetti, 2000). Por tanto, se considera que representa una forma de afrontamiento ante la adversidad (afrontamiento resiliente), promoviendo el uso de diferentes estrategias tanto conductuales como cognitivas (Sinclair \& Wallston, 2004). Según Sinclair \& Wallston (2004), los individuos que mostraron un patrón de afrontamiento resiliente tendieron a usar con una frecuencia mayor la reevaluación de la situación, así como la solución activa de las dificultades de la vida. Así, diferentes estudios llevados a cabo durante la pandemia por Covid-19, han evidenciado que contar con un alto nivel de afrontamiento resiliente se asociaba a mayores índices de salud mental (Li et al., 2021); se relacionaba con una mayor calidad de vida (Keener et al., 2021); y amortiguaba los efectos nocivos asociados al aislamiento percibido en este periodo (Salah et al., 2021). Por tanto, se podría considerar al afrontamiento resiliente como un recurso personal ante eventos estresantes, como puede ser la pandemia, pues dependiendo de la forma en que las personas se enfrentan a este tipo de eventos, tendrán una mayor o menor probabilidad de desarrollar problemas asociados a este tipo de circunstancias.

En vistas a lo señalado por la literatura previa revisada hasta ahora, por un lado, encontramos al FoMO como una de las consecuencias indeseables asociadas a este periodo de pandemia y, por otro, al afrontamiento resiliente como un recurso personal protector ante eventos vitales estresantes como los que la sociedad está viviendo actualmente. Conocer factores que supongan un factor protector añadido ante consecuencias indeseables asociadas a eventos vitales estresantes, como lo es el FoMO, podría servir para potenciar los efectos del afrontamiento resiliente, en aquellos casos cuyo nivel inicial sea alto, o para amortiguar las consecuencias negativas asociadas a un bajo nivel de afrontamiento resiliente.

En este sentido, algunos autores han apuntado al importante papel protector de las necesidades psicológicas básicas. Desde la Teoría de la Autodeterminación (Deci \& Ryan, 1985) el FoMO ha sido entendido como una experiencia emocional que surge cuando no se satisfacen determinadas necesidades psicológicas. Según esta teoría, la salud psicológica y la autorregulación emocional se relaciona con la satisfacción de tres necesidades: autonomía personal, sentimientos de afinidad y conexión social, y competencia para participar en el mundo. Diversos estudios han señalado que existe una relación negativa entre tener satisfechas estas necesidades básicas y el nivel de FoMO (Przybylski et al., 2013; Xie et al., 2018). Partiendo de esta teoría, diversos estudios han relacionado las dimensiones de Inteligencia Emocional, y en especial, la (auto)regulación emocional con estas necesidades básicas. Específicamente, entre los procesos centrales más relacionados con una mayor autonomía y un funcionamiento pleno, así como, con más y mejores relaciones sociales y sentimientos de competencia o autoeficacia se encontró la regulación emocional (Roth et al., 2019). La regulación emocional, que puede ser definida como la habilidad para gestionar o regular las emociones (tanto negativas como positivas) propias y de los demás (Mayer et al., 2016), ha mostrado un papel importante en el afrontamiento de situaciones ansiógenas y estresantes, repercutiendo de una forma positiva al propio bienestar (Limonero et al., 2006; Liniana \& Long, 2002). En este sentido, el estudio llevado a cabo por Sun et al. (2021) durante el periodo de pandemia, evidenciaron que contar con bajos niveles de regulación emocional se relacionaba con mayores índices de depresión y emociones negativas. A su vez, una de las estrategias de regulación 
emocional conocida como reevaluación cognitiva, ha sido relacionada positivamente con el nivel de felicidad (Gutiérrez-Cobo et al., 2021), así como se ha relacionado negativamente con el grado de ansiedad (Muñoz-Navarro et al., 2021) a lo largo de este periodo. Por tanto, podría considerarse la regulación emocional como un factor protector frente a consecuencias indeseables asociadas a la pandemia. Teniendo en cuenta que otra de las consecuencias asociadas a este periodo ha sido un aumento del fenómeno FoMO, resulta de interés conocer su relación con la regulación emocional. En este sentido, solo un estudio ha analizado la relación entre los niveles autocontrol (como una dimensión de un constructo más amplio) y el FoMO. Así, Kartol y Gündoğan (2020) en una investigación en la que participaron 370 sujetos de 18 a 30 años, encontraron que existe una relación negativa entre ambas variables, de forma que podría considerarse el autocontrol como un factor protector frente al miedo a perderse algo. Pese a que este estudio no hace alusión de forma independiente al componente regulación emocional, muestra indicios de cómo este constructo podría repercutir positivamente reduciendo los niveles de FoMO en la población.

Por tanto, atendiendo a lo señalado por la literatura descrita, existe evidencia que apunta al potencial papel protector que ejercen recursos personales como el afrontamiento resiliente y la regulación emocional, frente a eventos vitales estresantes, como es el periodo de pandemia que desde hace más de un año se vive en todo el mundo. Sin embargo, poco se conoce actualmente sobre estos recursos personales potencialmente protectores frente a una de las consecuencias fuertemente asociada a este periodo de pandemia, el FoMO. Explorar la acción conjunta de este afrontamiento resiliente junto a la regulación emocional sobre el FoMO, podría ser una gran oportunidad para conocer si combinan sus efectos entre sí, ejerciendo con ello, efectos protectores frente al FoMO.

Por tanto, los objetivos de este estudio fueron: (1) analizar la relación entre el afrontamiento resiliente y el FoMO; y (2) investigar el posible efecto moderador de la regulación emocional sobre dicha relación. Hasta donde sabemos, la regulación emocional no ha sido estudiada previamente como moderador entre el afrontamiento resiliente y el FoMO. Sin embargo, en vistas a los hallazgos anteriormente citados, es tentativo considerar que la regulación emocional podría, de algún modo, moderar la relación entre ambas variables, dado que los individuos con mayores niveles de regulación tienden a experimentar menos angustia psicológica y son capaces de mitigar de algún modo la influencia de pensamientos y sentimientos negativos que pueden surgir en situaciones estresantes (Domínguez-García \& Fernández-Berrocal, 2018), como puede ser la pandemia por Covid-19 que estamos viviendo en la actualidad.

En relación a los objetivos propuestos, esperamos encontrar que el afrontamiento resiliente se asocie negativamente con el FoMO (Hipótesis 1). Por otra parte, dado que la regulación emocional ayuda a regular las experiencias emocionales propias, también se espera que esta se vincule positivamente con el afrontamiento resiliente (Hipótesis 2). Por último, dado que tener un nivel más alto de regulación emocional puede ayudar a regular las emociones negativas relacionadas con el aislamiento social experimentado desde el inicio del periodo de pandemia, por un lado, se espera una relación negativa entre la regulación emocional y el FoMO (Hipótesis 3), e hipotetizamos que la regulación emocional ejercerá un papel moderador en la asociación entre el afrontamiento resiliente y el FoMO (Hipótesis 4). De esta forma, se espera que la interacción conjunta de la regulación emocional y el afrontamiento resiliente ejerzan un papel protector frente al miedo a perderse algo. Esto es, se hipotetiza que a altos niveles de afrontamiento resiliente y altos niveles de regulación emocional la probabilidad de presentar miedo a perderse algo sea menor.

\section{MÉTODO}

\section{Diseño y participantes}

Siguiendo un diseño transversal, se realizó un estudio correlacional de tipo descriptivo-exploratorio. La muestra fue de 146 participantes pertenecientes a la población general española (74\% mujeres), con edades comprendidas entre los 18 y los 25 años ( $M=19.62 ; D T=2.01)$. Estos datos, así como otros relativos al tipo de ocupación y al curso académico de los de los participantes en la actualidad, aparecen resumidos en la Tabla 1. 
Tabla 1. Estadísticos descriptivos de las variables edad, sexo, tipo de ocupación y curso académico de los estudiantes

\begin{tabular}{lll}
\hline & Media & D.T. \\
\hline Edad & 19.62 & 2.01 \\
\hline & $\mathrm{N}$ & $\%$ \\
\hline Sexo & & \\
Hombre & 38 & 26 \\
Mujer & 108 & 74 \\
\hline Ocupación actual & & \\
Estudiante grado medio/superior & 13 & 8.9 \\
Estudiante grado universitario & 121 & 82.88 \\
Estudiante máster & 10 & 6.85 \\
Estudiante bachillerato & 1 & .68 \\
Enfermero/a & 1 & .68 \\
\hline Curso académico estudiantes & & \\
Primer curso & 115 & 79.31 \\
Segundo curso & 14 & 9.66 \\
Tercer curso & 5 & 3.45 \\
Cuarto curso & 8 & 5.52 \\
Quinto curso & 1 & .69 \\
Sexto curso & 2 & 1.38 \\
\hline
\end{tabular}

NOTA: $\mathrm{N}=145$ en Curso Académico de los Estudiantes

\section{Instrumentos}

A continuación, se describirán los instrumentos que han sido utilizados en el presente estudio. Resulta importante aclarar previamente que los enunciados de todos ellos fueron adaptados con el fin de que fuesen respondidos atendiendo al periodo que comprende desde el inicio de la pandemia por Covid-19 hasta la actualidad.

Cuestionario sociodemográfico: Para la recogida de información sociodemográfica se desarrolló un cuestionario autoadministrado, en el que los participantes debían responder a diferentes cuestiones: sexo, edad, tipo de ocupación en la actualidad y curso académico.

Fear of missing out (FoMO; Przybylski et al., 2013). Para el presente estudio, se utilizó el cuestionario FoMO en su adaptación española (Gil et al., 2015), un cuestionario autoadministrado que evalúa las preocupaciones y miedos que pueden experimentar los sujetos al encontrarse desconectados de las experiencias de su contexto social extendido. Cuenta con 10 ítems que se valoran en una escala tipo Likert de 5 puntos, que va de 1 (nada) a 5 (mucho). Se considera que, a mayor puntuación, mayor miedo presenta el individuo a perderse algo. La escala ha demostrado adecuadas propiedades psicométricas en cuanto a fiabilidad, validez y consistencia interna en estudios previos $(\alpha=.85$; Gil et al., 2015).

Escala de Inteligencia Emocional de Wong y Law (WLEIS; Wong \& Law, 2002). Para el presente estudio, se ha utilizado el cuestionario WLEIS en su adaptación española (WLEIS-S; Extremera et al., 2019). Este instrumento autoadministrado cuenta con 16 ítems que evalúan las 4 dimensiones de la Inteligencia Emocional que fueron descritas por Salovey y Mayer (2007): reconocimiento y valoración de las emociones de los demás, expresión y valoración de las emociones propias, regulación emocional y uso de la emoción. El presente estudio únicamente se ha centrado en la subescala Regulación Emocional que cuenta con cuatro ítems. Las respuestas han de darse utilizando una escala tipo Likert de 7 puntos que va de 1 (totalmente en desacuerdo) a 7 (totalmente de acuerdo). Puntuaciones más altas son consideradas como niveles mayores de Regulación Emocional. En cuanto a las propiedades psicométricas de la escala adaptada, Extremera et al. (2019) encontraron que son adecuadas para la subescala de Regulación Emocional $(\alpha=.84)$. 
Brief Resilience Scale (BRS; Smith et al., 2008). Para el presente estudio se ha utilizado el cuestionario BRS en su version española (Rodríguez-Rey et al., 2016). Este instrumento evalúa el Afrontamiento Resiliente en población adulta. Contiene 6 ítems, algunos directos y otros inversos, que se responden siguiendo una escala tipo Likert de 5 puntos que va de 1 (totalmente en desacuerdo) a 5 (totalmente de acuerdo). Puntuaciones altas son indicativo de una mayor habilidad de Afrontamiento Resiliente. Esta escala cuenta con adecuadas propiedades psicométricas en población española según estudios previos ( $\alpha=.83$; Rodríguez-Rey et al., 2016).

\section{Procedimiento}

Para la realización de la presente investigación, se contó con la aprobación del Comité de Ética e Investigación de la Universidad de Málaga (62-2016-H). La recogida de datos se llevó a cabo a través de cuestionarios autoadministrados vía online, siguiendo un muestreo no probabilístico por conveniencia. Todos los participantes fueron informados sobre el objetivo del estudio, así como del cumplimento por parte del mismo de todas las normas éticas y, antes de comenzar con la contestación del cuestionario, dieron su consentimiento informado para participar. A su vez, se les informó de la opción de poder abandonarlo en caso no desear continuar participando en el estudio.

\section{Análisis de datos}

Para el análisis de los datos recogidos en el estudio se utilizó el programa SPSS v. 25.0. (IBM Company, 2017) y la macro de PROCESS (Hayes, 2018).

En primer lugar, se realizó un análisis de estadísticos descriptivos de las variables sociodemográficas de la muestra. Para este análisis descriptivo, en el caso de variables cualitativas se llevó a cabo el cálculo de la frecuencia y porcentaje; mientras que en variables cuantitativas se calculó la media y la desviación típica. A su vez, se realizó un análisis de la consistencia interna de los instrumentos (Alfa de Cronbach).

Posteriormente se llevaron a cabo análisis de correlaciones bivariadas con el fin de conocer las relaciones entre las variables principales y así comprobar las Hipótesis 1, 2 y 3 . Por último, para explorar el posible papel moderador de la regulación emocional en la asociación entre el afrontamiento resiliente y el FoMO, se empleó la macro de PROCESS (modelo 1; Hayes, 2018). Así, este análisis se utilizó para comprobar la Hipótesis 4.

\section{RESULTADOS}

\section{Análisis descriptivos}

En la Tabla 2, aparecen las consistencias internas, así como los estadísticos descriptivos de las puntaciones obtenidas en las principales variables objeto de estudio.

Tabla 2. Análisis descriptivo y consistencias internas de las principales variables objeto de estudio

\begin{tabular}{llll}
\hline & $\alpha$ & Media & D.T. \\
\hline Afrontamiento Resiliente & .82 & 17.33 & 4.87 \\
\hline Miedo a Perderse Algo (FoMO) & .73 & 22.10 & 5.42 \\
\hline Regulación Emocional & .87 & 4.63 & 1.29 \\
\hline
\end{tabular}

\section{Análisis de correlaciones}

El coeficiente de correlación de Pearson evidenció relaciones significativas entre las diferentes variables. Por un lado, tal y como se esperaba, se encontró una correlación negativa entre la regulación emocional y el FoMO. Por otra 
parte, se mostró que existe una correlación positiva entre la regulación emocional y el afrontamiento resiliente. Por último, se encontró una correlación negativa entre el afrontamiento resiliente y el FoMO. Los estadísticos asociados a estos resultados pueden verse en la Tabla 3.

Tabla 3. Correlación de Pearson $\left(r_{x y}\right)$ de las principales variables objeto de estudio

\begin{tabular}{lll} 
& FoMO & Afrontamiento Resiliente \\
\hline \multicolumn{2}{l}{ Miedo a Perderse Algo (FoMO) } \\
\hline \multicolumn{2}{l}{ Afrontamiento Resiliente } & $-.25^{* *}$ \\
\hline Regulación Emocional & $-.19^{*}$ & $.45^{* * *}$ \\
\hline & NOTA: ${ }^{*} \mathrm{p}<.05 ;^{* *} \mathrm{p}<.01 ;{ }^{* * *} \mathrm{p}<.001$
\end{tabular}

\section{Análisis de moderación}

Para explorar el posible efecto moderador de la regulación emocional sobre la relación entre afrontamiento resiliente y FoMO, se utilizó el modelo 1 de la macro de PROCESS de Hayes (2018). En este modelo, el afrontamiento resiliente se introdujo como variable independiente, el FoMO como variable dependiente y la regulación emocional como variable moderadora. El sexo, la edad y la ocupación actual se ingresaron como covariables. Los resultados de estos análisis se presentan en la Tabla 4. Los estadísticos señalaron un efecto significativo del afrontamiento resiliente sobre el FoMO ( $b=-.26 ; 95 \% / C:-.46$ a $-.05, t=-2.42, p<.05)$, siendo el tamaño del efecto $\left(f^{2}=.12\right)$ mediante G*Power 3.1.9.7. entre pequeño (.02) y mediano (.15) según Cohen. A su vez, se encontró un efecto significativo de la interacción afrontamiento resiliente $\mathrm{x}$ regulación emocional, sobre el miedo a perderse algo (B=-.14, $95 \%$ /C:-.26 a $-.02, t=-2.23, p<.05)$.

Tabla 4. Efecto moderador de la Regulación Emocional sobre el Afrontamiento Resiliente

\begin{tabular}{|c|c|c|c|c|c|c|}
\hline & \multirow[t]{2}{*}{$\beta$} & \multirow[t]{2}{*}{ SE b } & \multirow[t]{2}{*}{$\mathrm{R}^{2}$} & \multirow[t]{2}{*}{$\Delta \mathrm{R}^{2}$} & \multicolumn{2}{|l|}{ IC del 95\% } \\
\hline & & & & & Límite inferior & Límite superior \\
\hline FoMO & & & $.11^{*}$ & & & \\
\hline Constante & $20.57 * * *$ & 5.05 & & & 10.59 & 30.57 \\
\hline Sexo & .64 & 1.06 & & & -1.46 & 2.74 \\
\hline Edad & .06 & .24 & & & -.44 & .55 \\
\hline Ocupación actual & -.13 & .17 & & & -.47 & .21 \\
\hline$A R$ & $-.26^{*}$ & .11 & & & -.46 & -.05 \\
\hline RE & -.45 & .38 & & & -1.20 & .30 \\
\hline$A R \times R E$ & $-.14^{*}$ & .06 & & $.03 *$ & -.26 & -.02 \\
\hline
\end{tabular}

Como puede observarse en la Figura 1, la relación negativa entre el miedo a perderse algo y el afrontamiento resiliente fue significativa a niveles altos de regulación emocional $(B=-.43,95 \% / C:-.72$ a $-.16, t=-3.07, p<.01)$, mientras que a niveles bajos de regulación emocional la relación no fue significativa ( $B=-.08,95 \%$ IC:-.32 a .17, $t=-.61, p=.54)$. 


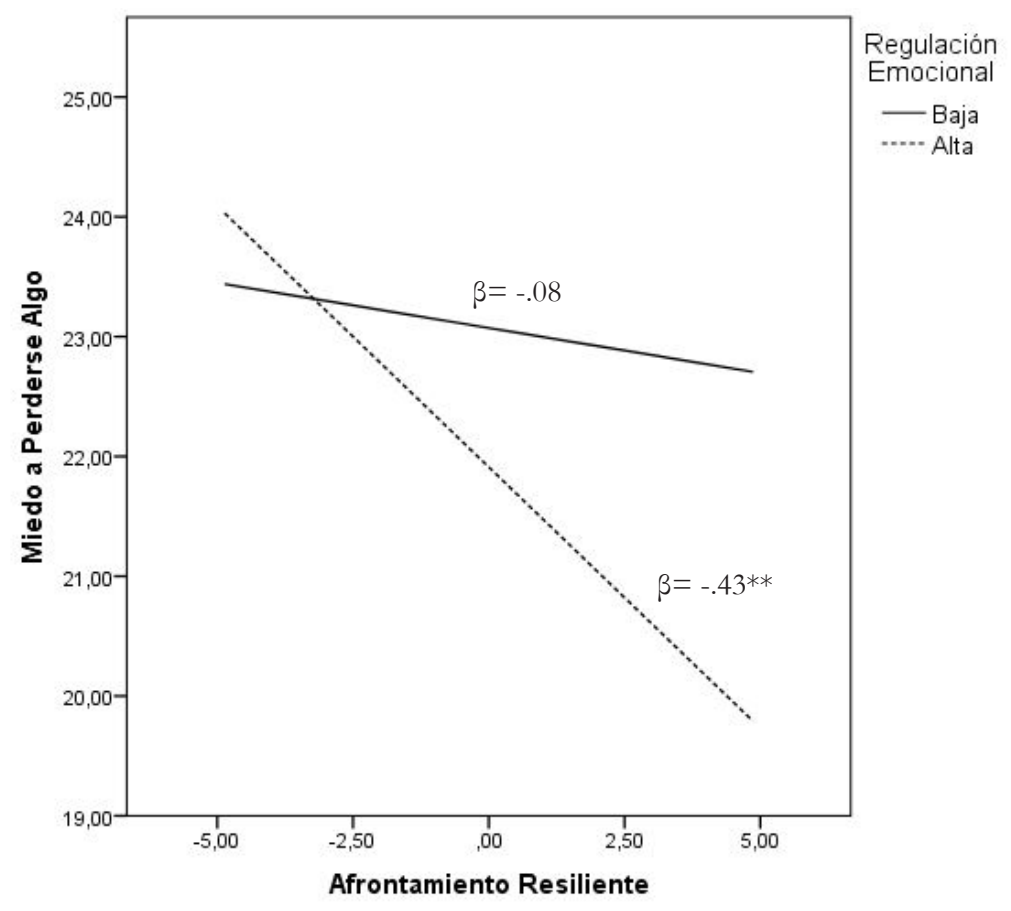

Figura 1. El papel moderador de la Regulación Emocional en la relación entre el FoMO y el Afrontamiento Resiliente NOTA: $* * p<.01$

\section{DISCUSIÓN}

El propósito principal de este estudio fue explorar la relación entre el afrontamiento resiliente y el FoMO, e investigar el posible efecto moderador de la regulación emocional sobre dicha relación en periodo de pandemia por Covid-19. Como se ha señalado anteriormente, existe evidencia sobre el papel protector que, ante eventos vitales estresantes como lo está siendo el actual periodo de pandemia, ejercen de manera individual el afrontamiento resiliente (Keener et al., 2021; Li et al., 2021; Salah et al., 2021) y la regulación emocional (Gutirrez-Cobo et al., 2021; Limonero et al., 2006; Liniana \& Long, 2002; Muñoz-Navarro et al., 2021; Sun et al., 2021). Sin embargo, el presente estudio se centra en conocer si ambos recursos personales ejercen un papel protector frente a una de las consecuencias nocivas asociadas a este periodo, el FoMO. Hasta donde sabemos, es la primera investigación que analiza el efecto de interacción de ambos constructos sobre el miedo a perderse algo en una muestra de jóvenes adultos. A continuación, se discutirán los resultados obtenidos en el presente estudio.

De acuerdo a los análisis de correlación, los resultados de este estudio confirman las hipótesis inicialmente propuestas en la dirección esperada. Por un lado, se ha podido confirmar la Hipótesis 1, al haberse encontrado que las personas que experimentan un mayor miedo a perderse algo, presentan un menor afrontamiento resiliente. Revisando la literatura, no se han encontrado estudios que analicen la relación entre ambas variables. Sin embargo, en contraste ante este hallazgo, nos basaremos en lo que dice la literatura respecto al efecto protector que ejerce el afrontamiento resiliente ante consecuencias indeseables asociadas a eventos estresantes. Así, en tiempos de pandemia como el actual, el afrontamiento resiliente ha sido considerado como un factor protector ante consecuencias negativas derivadas de este periodo, como son la ansiedad o la depresión (Sánchez et al., 2021), así como frente al malestar emocional (Viejo et al., 2020). A su vez, dentro de población estudiante joven adulta, se ha encontrado que aquellos con un menor afrontamiento resiliente sufrían un mayor impacto psicológico y académico derivados del confinamiento (Lozano-Díaz et al., 2020). Teniendo en cuenta que el FoMO se postula como una de las consecuencias no deseables de este periodo, es tentativo pensar que el afrontamiento resiliente ejerciera un papel protector 
ante este problema, al compartir este afrontamiento elementos relacionados con la satisfacción vital, como son una actitud positiva hacia la vida y el optimismo (Cazalla-Luna \& Molero, 2016). Así, una persona resiliente, afronta las situaciones estresantes de una forma satisfactoria, sobreponiéndose ante la adversidad, reduciéndose así significativamente las consecuencias negativas asociadas a este tipo de circunstancias, como podría ser el FoMO.

Otra de las hipótesis que nos marcamos inicialmente (Hipótesis 2) anticipaba una relación positiva entre el afrontamiento resiliente y la regulación emocional. Nuevamente, esta hipótesis se ha confirmado en la dirección esperada. Además, la literatura previa avala este resultado (Limonero et al., 2012; Yuan, 2021). Una posible explicación a esta asociación la da el Resilience Portfolio Model (Grych et al., 2015). Según esta teoría, las fortalezas que facilitan la resiliencia de las personas frente a situaciones adversas se pueden agrupar en tres dominios: fortalezas interpersonales, i.e. características individuales que fomentan el desarrollo y mantenimiento de relaciones sociales cercanas; las fortalezas de creación de significados, que se refieren a la capacidad personal de dar sentido a los eventos difíciles que suceden en la vida, manteniendo coherencia entre los sucesos, las creencias y los valores; y, por último, las fortalezas regulatorias, esto es, la regulación emocional frente a los contratiempos de la vida. Por tanto, atendiendo a esta teoría, la regulación emocional se considera como uno de los factores que facilitaría de forma directa el afrontamiento resiliente, pues parece favorecer una mayor comprensión y regulación de las emociones negativas asociadas a eventos estresantes.

Por su parte, también se ha podido confirmar la tercera hipótesis. Así, nuestros resultados sugieren que contar con un mayor nivel de regulación emocional como recurso personal, conduce a experimentar menores niveles de miedo a perderse algo. Resultados de estudios previos van en consonancia con este resultado. Así, una de las conclusiones del estudio llevado a cabo por Throuvala et al. (2019) fue que la falta de regulación emocional amplifica el uso de plataformas sociales tipo Snapchat, así como induce la aparición de otros problemas emocionales, como el FoMO que, a su vez, refuerza patrones compulsivos de uso de redes sociales. Otros estudios han asociado el FoMO a relaciones sociales pobres o de poca calidad, lo que a su vez se relaciona con la falta de regulación emocional (Przyblski et al., 2013; Ryan \& Deci, 2000). Por su parte, Kartol \& Gündoğan (2020) en su estudio encontraron que existe una relación negativa entre el FoMO y el autocontrol. Diversos autores aportan una explicación a esta asociación basándose en la Teoría de la Autodeterminación (Deci \& Ryan, 1985). Así, Przyblski et al. (2013) apuntan que una menor satisfacción con las necesidades psicológicas básicas de competencia para participar en el mundo, sentimientos de afinidad, autonomía personal y de conexión social, se asocian con una mayor vulnerabilidad para la desregulación emocional, con el consiguiente riesgo de desarrollar FoMO. Atendiendo a las características de este periodo de pandemia, en el que necesidades psicológicas básicas como los sentimientos de conexión social o de competencia para participar en el mundo, han podido verse amenazadas, resulta tentador pensar que pudieran haber supuesto un factor de riesgo a desarrollar problemas como el FoMO, pues de algún modo las personas no han podido regular sus emociones de una forma satisfactoria.

Por otra parte, en relación a la acción conjunta de la regulación emocional y el afrontamiento resiliente sobre el FoMO (Hipótesis 4), los análisis de moderación de este estudio han revelado un efecto de interacción de ambos constructos como forma de protección personal frente al FoMO. Como puede observarse en la Figura 1, aquellos sujetos que presentan altos niveles de regulación emocional y de afrontamiento resiliente, son los que tienen una menor probabilidad de presentar miedo a perderse algo. Además, las personas con un bajo nivel de regulación emocional muestran una alta probabilidad de desarrollar FoMO, independientemente de sus niveles de afrontamiento resiliente. La relación significativa entre el afrontamiento resiliente y el FoMO hallada únicamente a niveles altos de regulación emocional sugiere la importancia de contar con altas habilidades de regulación emocional dado que reduce significativamente la probabilidad de desarrollar FoMO. Por tanto, parece ser que contar con altos niveles de regulación emocional y afrontamiento resiliente pueden considerarse como factores protectores al reducir de forma conjunta la aparición del FoMO entre los jóvenes. De esta forma, pese a la importancia que supone el afrontamiento resiliente como forma de hacer frente de forma satisfactoria a eventos vitales estresantes, en relación al FoMO, este afrontamiento por sí solo parece no ser suficiente para su reducción, pues se necesita además contar con altos niveles de regulación emocional. Por tanto, se propone el desarrollo de habilidades de regulación emocional, pues potencia el efecto protector del afrontamiento resiliente ante problemáticas asociadas a eventos estresantes, como lo es el FoMO. 


\section{Limitaciones}

La presente investigación no está exenta de limitaciones. La principal está relacionada con el tamaño muestral y homogeneidad de la muestra. Por un lado, la muestra de este estudio está compuesta de un total de 146 participantes españoles de 18 a 25 años, de los que 108 corresponde al sexo femenino, por lo que podría existir un sesgo asociado al sexo en los resultados. Además, debido a las dificultades que han supuesto las diversas restricciones (e.g. de movilidad), la recogida de datos se realizó vía telemática y el tamaño muestral es relativamente pequeño. Asimismo, aunque no hay que descartar el tamaño del efecto pequeño como sugieren Funder y Ozer (2019), es necesario corroborar el modelo teórico de este estudio con muestras más numerosas para evitar posibles sesgos estadísticos y proporcionar estimaciones más precisas sobre el efecto encontrado. Por todo ello, sería necesario replicar estos resultados con un mayor tamaño muestral y mayor homogeneidad de sexos. Por otra parte, dado el corte transversal del estudio, se debería tener especial cuidado al realizar inferencias sobre el mismo, pues se trata de un diseño que impide efectuar relaciones de causalidad. Por tanto, sería importante que se realizasen futuros estudios de corte longitudinal con muestras que cuenten con más heterogeneidad (e. g. que aumente el número de la muestra, que ampliasen el rango de edad, que abarcase diferentes culturas,...). A su vez, dado que el FoMO se considera como un fenómeno complejo en el que intervienen distintos factores y que se asocia a diferentes problemas, resultaría necesario el estudio de otras variables y recursos que conduzcan a entender mejor este miedo, y que ayuden a favorecer su prevención.

Sin embargo, pese a las limitaciones de la presente investigación, los resultados encontrados aportan una serie de implicaciones, tanto prácticas como teóricas. Desde un punto de vista teórico, se ha intentado aumentar el conocimiento sobre el papel que ejercen diferentes factores con potencial carácter protector frente al FoMO, teniendo en cuenta el contexto de pandemia que desde Marzo de 2020 se vive en todo el mundo. Así, nuestros resultados permiten profundizar sobre diversas variables relevantes en este escenario, como son la regulación emocional y el afrontamiento resiliente, aumentando la comprensión sobre el papel que conjuntamente pueden ejercer sobre problemas como el FoMO.

Por su parte, desde un punto de vista aplicado, el efecto protector del afrontamiento resiliente junto a la regulación emocional sobre el miedo a perderse algo, podría ser tenido en cuenta de cara a la promoción de programas de prevención y de reducción del FoMO, evitando así los potenciales problemas y prácticas nocivas que pueden derivarse de este miedo. Un abordaje del fenómeno FoMO por medio del entrenamiento y desarrollo de recursos personales como la regulación emocional o el afrontamiento resiliente, no solo supondrán una mejora en este fenómeno, sino que tendrá una repercusión indirecta a la hora de ayudar a las personas a afrontar situaciones adversas y estresantes, como lo es la situación de alerta sanitaria que se está viviendo en la actualidad, evitando así consecuencias indeseables asociadas a este tipo de circunstancias.

\section{Conclusión}

El objetivo principal de la presente investigación ha sido conocer el papel protector conjunto de la regulación emocional y el afrontamiento resiliente frente al miedo a perderse algo (FoMO). Pese a que aún se necesita un cuerpo mayor de evidencia científica, los hallazgos de este estudio apuntan a los potenciales beneficios de entrenar y desarrollar ambos recursos y fortalezas con el fin de proteger de emociones negativas como el FoMO. Un abordaje preventivo, así como alertar de sus potenciales consecuencias nocivas, puede ser una forma útil de paliar esta problemática en la población, algo que cobra especial relevancia teniendo en cuenta el contexto de emergencia sanitaria en el que nos encontramos. 


\section{REFERENCIAS}

Adams, S. K., Williford, D. N., Vaccaro, A., Kisler, T. S., Francis, A., \& Newman, B. (2017). The young and the restless: Socializing trumps sleep, fear of missing out, and technological distractions in first-year college students. International Journal of Adolescence and Youth, 22, 337-348. https://doi.org/10.1080/02673843.2016.1181557

Alt, D. (2015). College students' academic motivation, media engagement and fear of missing out. Computers in Human Behavior, 49, 111-119. https://doi.org/10.1016/i.chb.2015.02.057

Ariza, A. C., Monzonís, N. C., Magaña, E. C., \& Méndez, V. G. (2021). Jóvenes y uso problemático de las tecnologías durante la pandemia: una preocupación familiar. Revista científica de educación y comunicación, 22, 1204-1204. https://doi.org/10.25267/Hachetetepe.2021.i22.1204

Baker, Z. G., Krieger, H., \& LeRoy, A. S. (2016). Fear of missing out: Relationships with depression, mindfulness, and physical symptoms. Issues in Psychological Science, 2(3), 275. https://doi.org/10.1037/tps0000075

Beyens, I., Frison, E., \& Eggermont, S. (2016). I don't want to miss a thing: Adolescents' fear of missing out and its relationship to adolescents' social needs, Facebook use, and Facebook related stress. Computers in Human Behavior, 64, 1-8. https://doi.org/10.1016/i.chb.2016.05.083

Błachnio, A., \& Przepiórka, A. (2018). Facebook Intrusion, fear of missing out, narcissism, and life satisfaction: A cross-sectional study. Psychiatry Research, 259, 514-519. https://doi.org/10.1016/j.psychres.2017.11.012.

Buglass, S. L., Binder, J. F., Betts, L. R., \& Underwood, J. D. M. (2017). Motivators of online vulnerability: The impact of social network site use and FoMO. Computers in Human Behavior, 66, 248-255. https://doi.org/10.1016/i. chb.2016.09.055.

Cazalla-Luna, N., y Molero, D. (2016). Inteligencia emocional percibida, disposición al optimismo-pesimismo, satisfacción vital y personalidad de docentes en su formación inicial. Revista de Investigación Educativa, 34(1), $241-258$. https://doi.org/10.6018/rie.34.1.220701

Deci, E. L., \& Ryan, R. M. (1985). Intrinsic motivation and selfdetermination in human behavior. Plenum.

Domínguez-García, E., \& Fernández-Berrocal, P. (2018). The association between emotional intelligence and suicidal behavior: A systematic review. Frontiers in Psychology, 9. https://doi.org/10.3389/fpsyg.2018.02380.

Dong, E., Du, H., \& Gardner, L. (2020). An interactive web-based dashboard to track COVID-19 in real time. The Lancet infectious diseases, 20(5), 533-534. https://doi.org/10.1016/S1473-3099(20)30120-1

Elhai, J. D., Levine, J. C., Dvorak, R. D., \& Hall, B. J. (2016). Fear of missing out, need for touch, anxiety and depression are related to problematic smartphone use. Computers in Human Behavior, 63, 509-516. https://doi.org/10.1016/j. chb.2016.05.079

Extremera, N., Rey, L., \& Sánchez- Álvarez, N. (2019). Validation of the Spanish version of the Wong Law emotional intelligence scale (WLEIS-S). Psicothema, 31, 94-100. https://doi.org/10.7334/psicothema2018.147

Fumagalli, E., Dolmatzian, M. B., \& Shrum, L. J. (2021). Centennials, FoMO, and loneliness: An investigation of the impact of social networking and messaging/VolP apps usage during the initial stage of the coronavirus pandemic. Frontiers in psychology, 12, 1-14. https://doi.org/10.3389/fpsyg.2021.620739 
Funder, D. C., \& Ozer, D. J. (2019). Evaluating Effect Size in Psychological Research: Sense and Nonsense. Advances in Methods and Practices in Psychological Science, 2(2), 156-168. https://doi.org/10.1177/2515245919847202Gao, J.,

Zheng, P., Jia, Y., Chen, H., Mao, Y., Chen, S., Wang, Y., Fu, H., \& Dai, J. (2020). Mental health problems and social media exposure during COVID-19 outbreak. PLOS ONE, 15(4), e0231924. https://doi.org/10.1371/journal.pone.0231924

Gil, F., Chamarro A. \& Oberst, U. (2015). Addiction to online social networks: A question of «Fear of Missing Out»? Journal of Behavioral Addictions, 4, 1-66. https://doi.org/10.1556/JBA.4.2015.Suppl.1

Gioia, F., Fioravanti, G., Casale, S., \& Boursier, V. (2021). The effects of the fear of missing out on people's social networking sites use during the COVID-19 pandemic: the mediating role of online relational closeness and individuals' online communication attitude. Frontiers in Psychiatry, 12, 1-11. https://doi.org/10.3389/fpsyt.2021.620442

Grych, J., Hamby, S., \& Banyard, V. (2015). The resilience portfolio model: Understanding healthy adaptation in victims of violence. Psychology of Violence, 5, 343-354. https://doi.org/10.1037/a0039671

Gutiérrez-Cobo, M. J., Megías-Robles, A., Gómez-Leal, R., Cabello, R., \& Fernández-Berrocal, P. (2021). Is It Possible to Be Happy during the COVID-19 Lockdown? A Longitudinal Study of the Role of Emotional Regulation Strategies and Pleasant Activities in Happiness. International Journal of Environmental Research and Public Health, 18(6), 1-11. https://doi.org/10.3390/ijerph18063211

Hayes, A.F. (2018). Introduction to Mediation, Moderation, and Conditional Process Analysis. A Regression-Based Approach (2 $\underline{a}$ ed.): Guilford Press.

Hayran, C., Anik, L., \& Gürhan-Canli, Z. (2021). A threat to loyalty: Fear of missing out (FoMO) leads to reluctance to repeat current experiences. PLOS ONE, 15(4), e0232318. https://doi.org/10.1371/iournal.pone.0232318

IBM Company (2017). IBM SPSS Statistics for Windows, Version 25.O.: IBM Corporation.

Kartol, A., \& Gündoğan, S. (2020). The mediating role of emotional intelligence in the relationship between the fear of missing out in social environments (FoMO) and depression. European journal of education studies, 6(11), 1-16. https://doi.org/10.5281/zenodo.3613903.

Keener, T. A., Hall, K., Wang, K., Hulsey, T., \& Piamjariyakul, U. (2021). Quality of Life, Resilience, and Related Factors of Nursing Students During the COVID-19 Pandemic. Nurse educator, 46(3), 143-148. https://doi.org/10.1097/ NNE.0000000000000969

Lasa, N. B., Benito, D. J., Manterola, A. G., Sánchez, J. P. E., García, J. L. P., \& Germán, M. Á. (2020). Las consecuencias psicológicas de la COVID-19 y el confinamiento. https://addi.ehu.eus/bitstream/handle/10810/45924/Consecuencias\%20psicol\%C3\%B3gicas\%20COVID-19\%20PR3\%20DIG.pdf?sequence=1\&isAllowed=y

Li, F., Luo, S., Mu, W., Li, Y., Ye, L., Zheng, X., \& Chen, X. (2021). Effects of sources of social support and resilience on the mental health of different age groups during the COVID-19 pandemic. BMC psychiatry, 21(1), 1-14. https://doi. org/10.1186/s12888-020-03012-1

Lima, C. K. T., de Medeiros Carvalho, P. M., Lima, I. D., de Oliveira Nunes, J. V., Saraiva, J. S., de Souza, R. I., Lima, C., \& Neto, M. L. R. (2020). The emotional impact of Coronavirus 2019-nCoV (new Coronavirus disease). Psychiatry research, 287, 112915. https://doi.org/10.1016/i.psychres.2020.112915 
Limonero, J. T., Tomás-Sábado, J., \& Fernández-Castro, J. (2006). Relación entre Inteligencia Emocional percibida y ansiedad ante la muerte en estudiantes universitarios. Ansiedad y Estrés, 12(2), 267-278. https://core.ac.uk/download/pdf/157852155.pdf

Limonero, J. T., Tomás-Sábado, J., Fernández-Castro, J., Gómez-Romero, M. J., \& Ardilla-Herrero, A. (2012). Estrategias de afrontamiento resilientes y regulación emocional: predictores de satisfacción con la vida. Behavioral Psychology/Psicología Conductual, 20(1), 183-196. https://cutt.ly/NnkMGEr

Liniana, L. J., \& Long, A. C. (2002). The role of emotionality and self-regulation in the appraisal-coping process: tests of direct and moderating effects. Journal of Applied Developmental Psychology, 23(4), 471-493. https://doi. org/10.1016/S0193-3973(02)00129-6

Lozano-Díaz, A., Fernández-Prados, J. S., Canosa, V. F., \& Martínez, A. M. M. (2020). Impactos del confinamiento por el COVID-19 entre universitarios: Satisfacción Vital, Resiliencia y Capital Social Online. International Journal of Socio/ogy of Education, 79-104. https://hipatiapress.com/hpjournals/index.php/rise/article/view/5925

Luthar, S. S., \& Cicchetti, D. (2000). The construct of resilience: implications for interventions and social policies. Development and Psychopathology, 12(4), 857-885. https://www.ncbi.nlm.nih.gov/pmc/articles/PMC1903337/

Mayer, J. D., Caruso, D. R., \& Salovey, P. (2016). The ability model of emotional intelligence: Principles and updates. Emotion review, 8(4), 290-300. https://doi.org/10.1177/1754073916639667

Muñoz-Navarro, R., Cano Vindel, A., Schmitz, F., Cabello, R., \& Fernández-Berrocal, P. (2021). Emotional disorders during the CoViD-19 outbreak in Spain: the role of sociodemographic risk factors and cognitive emotion regulation strategies. Health Education \& Behavior, 1-12. https://doi.org/10.1177/10901981211014101

Parrado-González, A., \& León-Jariego, J. C. (2020). COVID-19: Factores asociados al malestar emocional y morbilidad psíquica en población española. Revista Española de Salud Pública, 94(8), 1-16. https://www.mscbs.gob.es/biblioPublic/publicaciones/recursos propios/resp/revista cdrom/VOL94/ORIGINALES/RS94C 202006058.pdf

Polizzi, C., Lynn, S. J., \& Perry, A. (2020). Stress and coping in the time of Covid-19: Pathways to resilience and recovery. Clinical Neuropsychiatry, 17(2), 59-62. https://doi.org/10.36131/CN20200204

Przybylski, A. K., Murayama, K., DeHaan, C. R., \& Gladwell, V. (2013). Motivational, emotional, and behavioral correlates of fear of missing out. Computers in Human Behavior, 29(4), 1841-1848. https://doi.org/10.1016/i. chb.2013.02.014

Rodríguez-Rey, R., Alonso-Tapia, J., \& Hernansaiz-Garrido, H. (2016). Reliability and validity of the Brief Resilience Scale (BRS) Spanish Version. Psychological assessment, 28(5), 101- 110. https://doi.org/10.1037/pas0000191.

Roth, G., Vansteenkiste, M., \& Ryan, R. M. (2019). Integrative emotion regulation: Process and development from a self-determination theory perspective. Development and psychopathology, 31(3), 945-956. https://doi.org/10.1017/ S0954579419000403

Ryan, R., \& Deci, E. L. (2000). Self-determination theory and the facilitation of intrinsic motivation, social development, and well-being. American Psychologist, 55(1), 68-78. https://doi.org/10.1037/0003-066X.55.1.68.

Salah, A. B., DeAngelis, B. N., \& al'Absi, M. (2021). Resilience and the Role of Depressed and Anxious Mood in the Relationship Between Perceived Social Isolation and Perceived Sleep Quality During the COVID-19 Pandemic. International Journal of Behavioral Medicine, 28(3), 277-285. https://doi.org/10.1007/s12529-020-09945-x 
Salovey, P. \& Mayer, J. D., (2007). Manual de Inteligencia Emocional: Pirámide.

Sánchez, C. G. C., Cotrina-Aliaga, J. C., \& Aguinaga-Villegas, D. (2021). La resiliencia como factor fundamental en tiempos de Covid-19. Propósitos y Representaciones, 9(1), 1044. http://revistas.usil.edu.pe/index.php/pyr/article/ view/1044

Sandín, B., Valiente, R. M., García-Escalera, J., \& Chorot, P. (2020). Impacto psicológico de la pandemia de COVID-19: Efectos negativos y positivos en población española asociados al periodo de confinamiento nacional. Revista de Psicopatología y Psicología Clínica, 25(1). https://cutt.ly/GbXRtir

Sinclair, V. G., \& Wallston, K. A. (2004). The development and psychometric evaluation of the Brief Resilient Coping Scale. Assessment, 11, 94-101. https://doi.org/10.1177/1073191103258144

Smith, B.W., Dalen, J., Wiggins, K., Tooley, E., Christopher, P., \& Bernard, J. (2008). The brief resilience scale: assessing the ability to bounce back. International Journal of Behavioral Medicine, 15(3), 194-200. https://doi. org/10.1080/10705500802222972

Sun, H., Wang, S., Wang, W., Han, G., Liu, Z., Wu, Q., \& Pang, X. (2021). Correlation between emotional intelligence and negative emotions of front-line nurses during the COVID-19 epidemic: A cross-sectional study. Journal of clinical nursing, 30(3), 385-396. https://doi.org/10.1111/jocn.15548

Throuvala, M. A., Griffiths, M. D., Rennoldson, M., \& Kuss, D. J. (2019). Motivational processes and dysfunctional mechanisms of social media use among adolescents: A qualitative focus group study. Computers in Human Behavior, 93, 164-175. https://doi.org/10.1016/i.chb.2018.12.012

Viejo, J. M. P., Barbé, A. D., Pérez, M. D. M. R. B., \& Pérez, J. L. (2020). Resiliencia para la promoción de la salud en la crisis Covid-19 en España. Revista de Ciencias Sociales, 26(4), 52-63. https://dialnet.unirioja.es/servlet/articulo?codigo=7687026

Wegmann, E., Oberst, U., Stodt, B., \& Brand, M. (2017). Online-specific fear of missing out and Internet-use expectancies contribute to symptoms of Internet-communication disorder. Addictive Behaviors Reports, 5, 33-42. https:// doi.org/10.1016/i.abrep.2017.04.001

Wong, C. S., \& Law, K. S. (2002). The effects of leader and follower emotional intelligence on performance and attitude: An exploratory study. The Leadership Quarterly, 13(3), 243-274. https://doi.org/10.1016/S1048-9843(02)00099-1

Xie, X., Wang, Y., Wang, P., Zhao, F., \& Lei, L. (2018). Basic psychological needs satisfaction and fear of missing out: Friend support moderated the mediating effect of individual relative deprivation. Psychiatry Research, 268, 223228. https://doi.org/10.1016/i.psychres.2018.07.025

Yuan, Y. (2021). Mindfulness training on the resilience of adolescents under the COVID-19 epidemic: A latent growth curve analysis. Personality and Individual Differences, 172, 110560. https://doi.org/10.1016/ i.paid.2020.110560 